\title{
Weighted Aztec Diamond Graphs and the Weyl Character Formula
}

\author{
Georgia Benkart* \\ Department of Mathematics \\ University of Wisconsin \\ Madison, WI 53706 \\ e-mail: benkart@math.wisc.edu \\ Oliver Eng \\ Epic Systems Corporation \\ 5301 Tokay Blvd. \\ Madison, WI 53711 \\ e-mail: oeng@epicsystems.com
}

Submitted: Nov 19, 2002; Accepted: Jan 20, 2004; Published: Apr 2, 2004

MR Subject Classification: 52C20, 05B45, 17B10

Keywords: Aztec diamonds, domino tilings, Weyl character formula

\begin{abstract}
Special weight labelings on Aztec diamond graphs lead to sum-product identities through a recursive formula of Kuo. The weight assigned to each perfect matching of the graph is a Laurent monomial, and the identities in these monomials combine to give Weyl's character formula for the representation with highest weight $\rho$ (the half sum of the positive roots) for the classical Lie algebras.
\end{abstract}

Choose a positive integer $n$ and label the $2 n \times 2 n$ checkerboard matrix style. The Aztec diamond of order $n$ is the subset of this checkerboard consisting of the squares whose coordinates $(i, j)$ satisfy $|j-i| \leq n$ and $(n+1) \leq i+j \leq(3 n+1)$. Thus, in an Aztec diamond of order $n$ there will be $2 n$ rows having $2,4, \ldots, 2 n, 2 n, \ldots, 4,2$ squares from top to bottom, as in Figure 1. A domino covers two adjacent squares, and the number of domino tilings of the Aztec diamond of order $n$ is $2^{n(n+1) / 2}$ by [EKLP1, EKLP2]. Those

\footnotetext{
${ }^{*}$ Support from National Science Foundation grant \#DMS-9970119 is gratefully acknowledged.
} 
papers establish connections between domino tilings of Aztec diamonds and alternating sign matrices, which in turn are related to a host of topics - such as states in the "square ice" model, complete monotone triangles, and descending plane partitions (see for example, $[\mathrm{Br}])$.

A monotone triangle is a triangular array $T$ of positive integers which strictly increase from left to right along its rows and weakly increase left to right along all of its diagonals. When the bottom row consists of $1,2, \ldots$, as in the example below, then $T$ is said to be a complete monotone triangle.

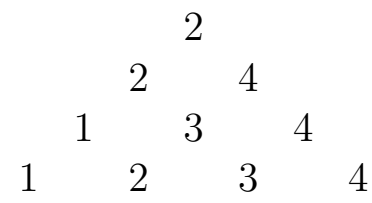

As shown in [EKLP1, Sec. 4], the number $A D(n)$ of domino tilings of the Aztec diamond of order $n$ is given by $A D(n)=\sum_{T \in \mathcal{T}_{n+1}} 2^{\vartheta(T)}$, where the sum is over the set $\mathcal{T}_{n+1}$ of complete monotone triangles $T$ with $n+1$ rows, and $\vartheta(T)$ is the number of entries in $T$ that do not occur in the row directly beneath it. In the above example $\vartheta(T)=1$. Section 5 of [EKLP2] connects these ideas with the representation theory of the complex general linear group $\mathrm{GL}_{n+1}$ (or equivalently of its Lie algebra $\mathfrak{g l}_{n+1}$ ). Let $V=\mathbb{C}^{n+1}$ and let $X=\Lambda^{2}(V)$, the second exterior power of $V$. Assume $a_{i}$ are positive integers satisfying $a_{1}<a_{2}<\cdots<a_{n+1}$. Consider the character

$$
g\left(x_{1}, \ldots, x_{n+1}\right):=\operatorname{Ch}\left(\Psi_{\underline{a}} \otimes \Lambda(X)\right)
$$

of the tensor product module $\Psi_{\underline{a}} \otimes \Lambda(X)$, where $\Psi_{\underline{a}}$ is the irreducible $\mathrm{GL}_{n+1}$-module with highest weight $\underline{a}=\left(a_{1}-1, a_{2}-2, \ldots, a_{n+1}-(n+1)\right)$ and $\Lambda(X)=\bigoplus_{j=0}^{n(n+1) / 2} \Lambda^{j}(X)$, the exterior algebra generated by $X$ (regarded as a $\mathrm{GL}_{n+1}$-module). In [EKLP2, Sec. 5], it is argued that $g(1,1, \ldots, 1)=\sum_{T} 2^{\vartheta(T)}$, where $T$ ranges over all monotone triangles with bottom row $a_{1}<a_{2}<\cdots<a_{n+1}$. The case $a_{i}=i$ for all $i=1, \ldots, n+1$ corresponds to the complete monotone triangles. However, $g(1,1, \ldots, 1)$ is also the dimension of the corresponding module, which in this particular case is the one-dimensional $\mathrm{GL}_{n+1}$-module $\Psi_{\underline{0}}$ with highest weight $\underline{0}=(0, \ldots, 0)$ tensored with $\Lambda(X)$. Thus,

$$
A D(n)=\sum_{T \in \mathcal{T}_{n+1}} 2^{\vartheta(T)}=g(1,1, \ldots, 1)=\operatorname{dim}\left(\Psi_{\underline{0}} \otimes \Lambda(X)\right)=1 \times 2^{n(n+1) / 2} .
$$

The purpose of this article is to establish a new connection between domino tilings of the Aztec diamond and the representation theory of all the classical Lie algebras. For this we specialize Stanley's weight labeling of the Aztec diamond graph and show that the specialized weight of a perfect matching of the graph corresponds to a Laurent monomial in Weyl's character formula for $\Psi_{\rho}$, the irreducible representation of $\mathfrak{s l}_{n+1}$ with highest weight $\rho$, where $\rho$ is half the sum of the positive roots. The number of times a given monomial occurs, which is the dimension of the weight space in the Lie sense, is precisely 
the number of matchings of a given weight. Thus, the perfect matchings of the Aztec diamond graph can be used to index a basis for $\Psi_{\rho}$. In a similar fashion, we show that perfect matchings on pairs of Aztec diamond graphs can be used to index a basis for $\Psi_{\rho}$ for the classical Lie algebras of types $\mathrm{B}_{n}, \mathrm{C}_{n}$, and $\mathrm{D}_{n}$. These Lie algebras were not considered in [EKLP1, EKLP2].

The Aztec diamond graph of order $n$ is the dual graph to the Aztec diamond of order $n$ in which the vertices are the squares and an edge joins two vertices if and only if the corresponding squares are adjacent in the Aztec diamond. A perfect matching on the Aztec diamond graph is a subgraph containing all the vertices such that each vertex has order exactly 1 . Identifying each edge in a perfect matching with a domino shows that the perfect matchings on the Aztec diamond graph are in bijective correspondence with the tilings of the Aztec diamond. See Figure 2 for a matching on the order 2 Aztec diamond graph.

It will be easier to work with the Aztec diamond graphs rotated 45 degrees counterclockwise to produce a figure such as Figure 3. Then one may locate an edge by the row $i$ and column $j$ that it lies in, where $i=1,2, \ldots, 2 n$ and $j=1,2, \ldots, 2 n$. Given an Aztec diamond graph of order $n$ called $A$, let $A_{N E}$ denote the Aztec diamond graph of order $n-1$ which contains the northeasternmost edge of $A$ in row 1 and column $2 n$, fitting snugly in the northeast corner of $A$. Similarly, define $(n-1)$-order Aztec diamond subgraphs $A_{N W}, A_{S W}$, and $A_{S E}$. Let $A_{\text {mid }}$ be the $(n-2)$-order Aztec diamond subgraph of $A$ lying directly in the middle, concentric with $A$.

For the rest of the paper, Aztec diamond graphs have edge weights. Figure 3 shows an Aztec diamond graph whose edges are weighted with integers. Given a matching $m$ of the Aztec diamond graph $A$, define the weight of the matching $\varpi(m)$ to be the product of the weights of all the edges in the matching. Then the weight of the Aztec graph $A$ is $\varpi(A)=\sum_{m} \varpi(m)$, the sum over all matchings of $A$. Using the tilted version of the Aztec diamond graph, Kuo $[\mathrm{K}]$ proved the following theorem:

Theorem 1 (Kuo) Let $A$ be a weighted Aztec diamond graph of order $n$. Also, let $\varpi_{N E}, \varpi_{N W}, \varpi_{S W}, \varpi_{S E}$ be the weights of the northeasternmost, northwesternmost, southwesternmost, and southeasternmost edges of $A$, respectively. Then

$$
\varpi(A)=\frac{\varpi_{S W} \cdot \varpi_{N E} \cdot \varpi\left(A_{N W}\right) \cdot \varpi\left(A_{S E}\right)+\varpi_{N W} \cdot \varpi_{S E} \cdot \varpi\left(A_{S W}\right) \cdot \varpi\left(A_{N E}\right)}{\varpi\left(A_{\text {mid }}\right)} .
$$

Stanley proposed the weight labeling displayed in Figure 6. We first learned about this labeling and the next theorem, which gives a product expression for the weight sum, from a talk by J. Propp $[\mathrm{P}]$. The method of proof outlined in the talk relied on "local transformations." (Compare also [C2] for related weight labelings.)

Theorem 2 Let $A$ be a weighted Aztec diamond graph of order $n$ with weight labeling as in Figure 6. Then 


$$
\varpi(A)=\prod_{1 \leq i \leq \ell \leq n}\left(y_{2 i-1} y_{2 \ell}+z_{2 i-1} z_{2 \ell}\right)
$$

Here we present an alternate proof based on Kuo's result.

Proof. When $n=1$, the Aztec diamond graph $A$ consists of one box with labels $y_{1}, z_{1}, y_{2}, z_{2}$ on its NW, NE, SE, SW edges respectively. There are two matchings, and the sum of their weights is $y_{1} y_{2}+z_{1} z_{2}$, so that the result holds in this case. When $n=2$, one may use Figure 5 to verify that the weighted sum is as follows:

$$
\begin{aligned}
\sum_{i=1}^{8} \varpi\left(m_{i}\right)= & y_{1}^{2} y_{2} y_{3} y_{4}^{2}+y_{1} y_{3} y_{4}^{2} z_{1} z_{2}+y_{1}^{2} y_{2} y_{4} z_{3} z_{4}+y_{1} y_{4} z_{1} z_{2} z_{3} z_{4} \\
& +z_{1}^{2} z_{2} z_{3} z_{4}^{2}+y_{3} y_{4} z_{1}^{2} z_{2} z_{4}+y_{1} y_{2} z_{1} z_{3} z_{4}^{2}+y_{1} y_{2} y_{3} y_{4} z_{1} z_{4} \\
= & \left(y_{1} y_{2}+z_{1} z_{2}\right)\left(y_{1} y_{4}+z_{1} z_{4}\right)\left(y_{3} y_{4}+z_{3} z_{4}\right) .
\end{aligned}
$$

Proceeding inductively, we obtain from Kuo's recursive theorem that

$$
\begin{aligned}
\varpi(A) & =\left(y_{1} y_{2 n}+z_{1} z_{2 n}\right) \frac{\prod_{1 \leq i \leq \ell \leq n-1}\left(y_{2 i-1} y_{2 \ell}+z_{2 i-1} z_{2 \ell}\right) \prod_{2 \leq p \leq r \leq n}\left(y_{2 p-1} y_{2 r}+z_{2 p-1} z_{2 r}\right)}{\prod_{\substack{2 \leq a \leq b \leq n-1\\
}}\left(y_{2 a-1} y_{2 b}+z_{2 a-1} z_{2 b}\right)} \\
& =\prod_{1 \leq i \leq \ell \leq n}\left(y_{2 i-1} y_{2 \ell}+z_{2 i-1} z_{2 \ell}\right) .
\end{aligned}
$$

By setting $y_{j}=1=z_{j}$ for all $j=1, \ldots, n$ in this expression, we see that the number of matchings, and hence the number $A D(n)$ of domino tilings of the Aztec diamond of order $n$, is $2^{n(n+1) / 2}$. In [EKLP1], four proofs of that result are presented. Ciucu [C1] has shown that $A D(n)=2^{n} A D(n-1)$, from which $A D(n)=2^{n(n+1) / 2}$ is an immediate consequence. In fact, Ciucu proves a more general recurrence for perfect matchings of cellular graphs.

Next we consider four different weight labelings of the Aztec diamond graph of order $n$, which are pictured in Figures 7, 8, 9, and 10. All are specializations of the Stanley labeling.

Corollary 1 Let $P$ be an Aztec diamond graph of order $n$ with

Weight Labeling A,

$$
\begin{array}{cc}
y_{2 i-1}=x_{i}^{-1} & y_{2 i}=x_{i+1} \\
z_{2 i-1}=x_{i} & z_{2 i}=x_{i+1}^{-1},
\end{array}
$$


for $1 \leq i \leq n$. Then

$$
\varpi(P)=\prod_{1 \leq i<j \leq n+1}\left(x_{i} x_{j}^{-1}+x_{j}^{-1} x_{i}\right)
$$

Proof. From the theorem we obtain

$$
\varpi(P)=\prod_{1 \leq i \leq \ell \leq n}\left(x_{i}^{-1} x_{\ell+1}+x_{i} x_{\ell+1}^{-1}\right)=\prod_{1 \leq i<j \leq n+1}\left(x_{i}^{-1} x_{j}+x_{i} x_{j}^{-1}\right)
$$

upon setting $j=\ell+1$.

Corollary 2 Let $P$ be an Aztec diamond graph of order $n$ with Weight Labeling B,

$$
\begin{array}{crr} 
& x_{0}=1 & \\
y_{2 i-1}=x_{i-1}^{-1} & & y_{2 i}=x_{i} \\
z_{2 i-1}=x_{i-1} & & z_{2 i}=x_{i}^{-1},
\end{array}
$$

for $1 \leq i \leq n$. Then

$$
\varpi(P)=\prod_{1 \leq i<j \leq n}\left(x_{i} x_{j}^{-1}+x_{i}^{-1} x_{j}\right) \prod_{1 \leq k \leq n}\left(x_{k}+x_{k}^{-1}\right) .
$$

Proof.

$$
\begin{aligned}
\varpi(P) & =\prod_{1 \leq i \leq j \leq n}\left(x_{i-1}^{-1} x_{j}+x_{i-1} x_{j}^{-1}\right) \\
& =\prod_{1 \leq j \leq n}\left(x_{j}+x_{j}^{-1}\right) \prod_{1 \leq i<j \leq n}\left(x_{i}^{-1} x_{j}+x_{i} x_{j}^{-1}\right) .
\end{aligned}
$$

Corollary 3 Let $P$ be an Aztec diamond graph of order $n$ with Weight Labeling $C$,

$$
\begin{array}{cc}
y_{2 i-1}=x_{i}^{-1} & y_{2 i}=x_{i}^{-1} \\
z_{2 i-1}=x_{i} & z_{2 i}=x_{i},
\end{array}
$$

for $1 \leq i \leq n$. Then

$$
\varpi(P)=\prod_{1 \leq i \leq j \leq n}\left(x_{i} x_{j}+x_{i}^{-1} x_{j}^{-1}\right)=\prod_{1 \leq k \leq n}\left(x_{k}^{2}+x_{k}^{-2}\right) \prod_{1 \leq i<j \leq n}\left(x_{i} x_{j}+x_{i}^{-1} x_{j}^{-1}\right) .
$$


Corollary 4 Let $P$ be an Aztec diamond graph of order $n$ with

Weight Labeling D,

$$
\begin{array}{cc}
y_{2 i-1}=x_{i}^{-1} & y_{2 i}=x_{i+1}^{-1} \\
z_{2 i-1}=x_{i} & z_{2 i}=x_{i+1},
\end{array}
$$

for $1 \leq i \leq n$. Then

$$
\varpi(P)=\prod_{1 \leq i<j \leq n+1}\left(x_{i} x_{j}+x_{i}^{-1} x_{j}^{-1}\right) .
$$

Suppose $\mathfrak{g}$ is a finite-dimensional simple complex Lie algebra corresponding to an irreducible root system $\Phi$. Let $\Phi^{+}$denote the positive roots, $W$ be the Weyl group, $l(w)$ be the length of an element $w \in W$, and let $\rho=\frac{1}{2} \sum_{\alpha \in \Phi^{+}} \alpha$. Let $\Psi_{\rho}$ denote the irreducible representation of $\mathfrak{g}$ with highest weight $\rho$. Applying the Weyl character and denominator formulas (as in $[\mathrm{FH}]$ or $[\mathrm{H}]$ for example), one sees that

$$
\begin{aligned}
\mathrm{Ch}\left(\Psi_{\rho}\right) & =\frac{\sum_{w \in W}(-1)^{l(w)} e^{w(2 \rho)}}{\sum_{w \in W}(-1)^{l(w)} e^{w(\rho)}}=\frac{\sum_{w \in W}(-1)^{l(w)}\left(e^{2}\right)^{w(\rho)}}{\sum_{w \in W}(-1)^{l(w)} e^{w(\rho)}} \\
& =\frac{\prod_{\alpha \in \Phi^{+}}\left(e^{2}\right)^{\alpha / 2}-\left(e^{2}\right)^{-\alpha / 2}}{\prod_{\alpha \in \Phi^{+}} e^{\alpha / 2}-e^{-\alpha / 2}}=\frac{\prod_{\alpha \in \Phi^{+}} e^{\alpha}-e^{-\alpha}}{\prod_{\alpha \in \Phi^{+}} e^{\alpha / 2}-e^{-\alpha / 2}} \\
& =\prod_{\alpha \in \Phi^{+}}\left(e^{\alpha / 2}+e^{-\alpha / 2}\right) .
\end{aligned}
$$

When the product is expanded, each factor contributes one of either $e^{\alpha / 2}$ or $e^{-\alpha / 2}$ to each term, so that each term in the sum contributes one to the dimension. Hence, the dimension of $\Psi_{\rho}$ is $2^{\left|\Phi^{+}\right|}$. The number of roots as well as a description of the positive roots for the classical Lie algebras are given in Figure 11. The vectors $\left\{\varepsilon_{1}, \varepsilon_{2}, \ldots, \varepsilon_{n}\right\}$ appearing in this table form an orthonormal basis of unit vectors with respect to the usual inner product in $\mathbf{R}^{n}$. Additional information about root systems can be found in $[\mathrm{B}]$ or $[\mathrm{H}]$.

Theorem 3 Let $P$ be an Aztec diamond graph of order $n$ with Weight Labeling A. Let $\Psi_{\rho}$ be the irreducible representation with highest weight $\rho$ for type $A_{n}$. Substituting $x_{i}=e^{\varepsilon_{i} / 2}$ for $i=1,2, \ldots, n+1$ in the weight labeling gives

$$
\varpi(P)=\operatorname{Ch}\left(\Psi_{\rho}\right)
$$

Proof. The theorem follows immediately from Corollary 1.

Similarly, we have the following theorems, whose results are summarized in Figure 12. 
Theorem 4 Let $P$ be an Aztec diamond graph of order $n$ with Weight Labeling B, and let $Q$ be an Aztec diamond graph of order $n-1$ with Weight Labeling D. Assume $\Psi_{\rho}$ is the irreducible representation with highest weight $\rho$ for type $B_{n}$. Substituting $x_{i}=e^{\varepsilon_{i} / 2}$ for $i=1,2, \ldots, n$ in the weight labelings for both Aztec diamond graphs gives

$$
\varpi(P) \varpi(Q)=\operatorname{Ch}\left(\Psi_{\rho}\right)
$$

Theorem 5 Let $P$ be an Aztec diamond graph of order $n$ with Weight Labeling $C$, and let $Q$ be an Aztec diamond graph of order $n-1$ with Weight Labeling A. Assume $\Psi_{\rho}$ be the irreducible representation with highest weight $\rho$ for type $C_{n}$. Substituting $x_{i}=e^{\varepsilon_{i} / 2}$ for $i=1,2, \ldots, n$ in the weight labelings for both Aztec diamond graphs gives

$$
\varpi(P) \varpi(Q)=\operatorname{Ch}\left(\Psi_{\rho}\right)
$$

Theorem 6 Let $P$ and $Q$ be Aztec diamonds graph of order $n-1$ with Weight Labeling $D$ and Weight Labeling A respectively. Let $\Psi_{\rho}$ be the irreducible representation with highest weight $\rho$ for type $D_{n}$. Substituting $x_{i}=e^{\varepsilon_{i} / 2}$ for $i=1,2, \ldots, n$ in the weight labelings for both Aztec diamond graphs gives

$$
\varpi(P) \varpi(Q)=\operatorname{Ch}\left(\Psi_{\rho}\right)
$$

The character records the dimensions of the weight spaces in an irreducible representation $\Psi_{\lambda}$ with highest weight $\lambda$. The weight space $\Psi_{\lambda}^{\mu}$ associated to the weight $\mu$ is a common eigenspace for a Cartan subalgebra $\mathfrak{h}$ of the simple Lie algebra, where $h \in \mathfrak{h}$ acts with eigenvalue $\mu(h)$. Thus, the character is given by

$$
\operatorname{Ch}\left(\Psi_{\lambda}\right)=\sum_{\mu} \operatorname{dim}\left(\Psi_{\lambda}^{\mu}\right) e^{\mu}
$$

The theorems above treat the special case where $\lambda=\rho$. Applying Theorem 3, we have

$$
\begin{aligned}
\sum_{\mu} \operatorname{dim}\left(\Psi_{\rho}^{\mu}\right) e^{\mu} & =\operatorname{Ch}\left(\Psi_{\rho}\right) \\
& =\varpi(P) \\
& =\sum_{\mu}\left|M(P)_{\mu}\right| e^{\mu},
\end{aligned}
$$

where $M(P)$ is the set of all perfect matchings of the Aztec diamond graph $P$ of order $n$ with Weight Labeling $\mathrm{A}$, and $M(P)_{\mu}$ is the set of all those matchings having weight $\mu$. By equating the coefficients of each monomial in the sum, we see that the set of matchings in $M(P)$ of weight $\mu$ is equinumerous with a set of basis vectors for the weight space 
$\Psi_{\rho}^{\mu}$. Thus, these matchings can be used to index a basis for that weight space. There are analogous interpretations of the other theorems using pairs of matchings of Aztec diamond graphs.

In this paper, we have indexed a basis of the irreducible representation with highest weight $\rho$ for the classical Lie algebras by the perfect matchings of the Aztec diamond graph. The matchings of other graphs (such as the ones associated to fortresses and dungeons in $[\mathrm{Y}]$, [C2], and [P2]) may have similar interesting Lie theoretic interpretations.

\section{Figures}
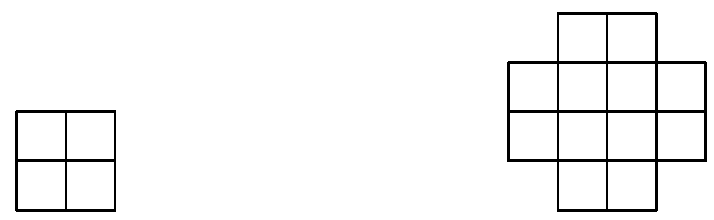

Figure 1: Aztec diamonds of order 1 and order 2
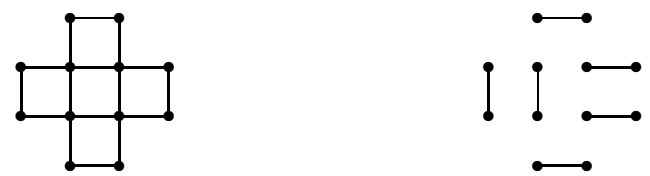

Figure 2: Aztec diamond graph of order 2 and a matching on it

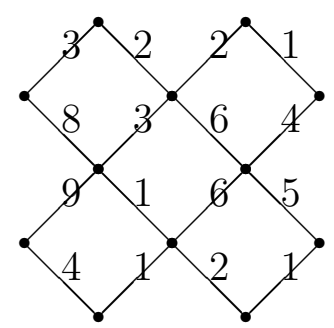

Figure 3: Aztec diamond graph rotated 45 degrees with integer edge weights 

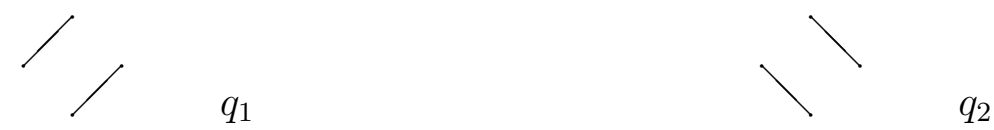

Figure 4: All matchings of the Aztec diamond graph of order 1
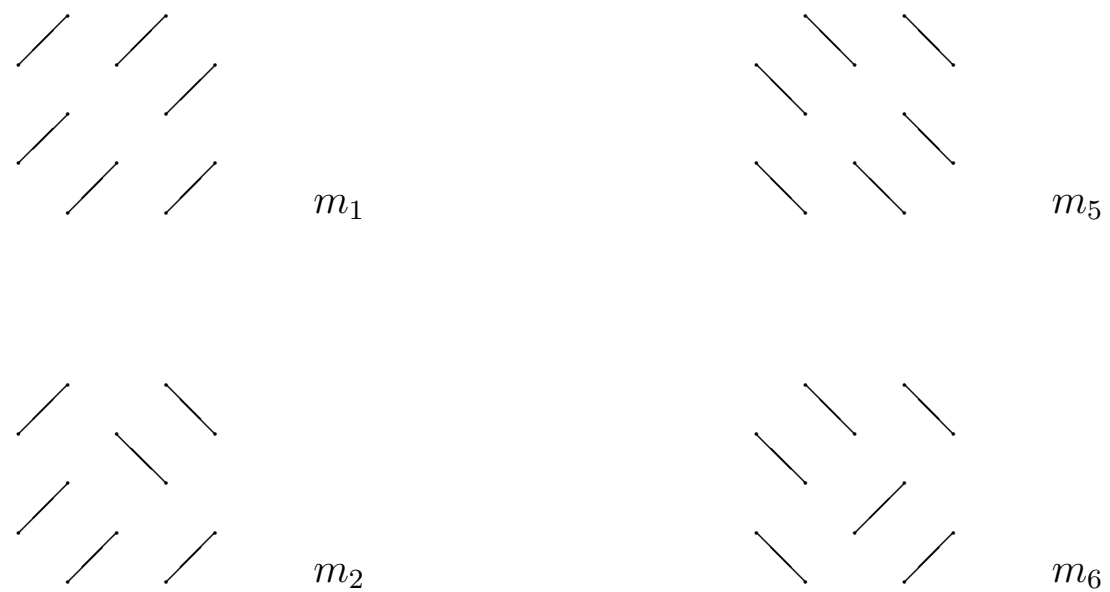

$m_{2}$
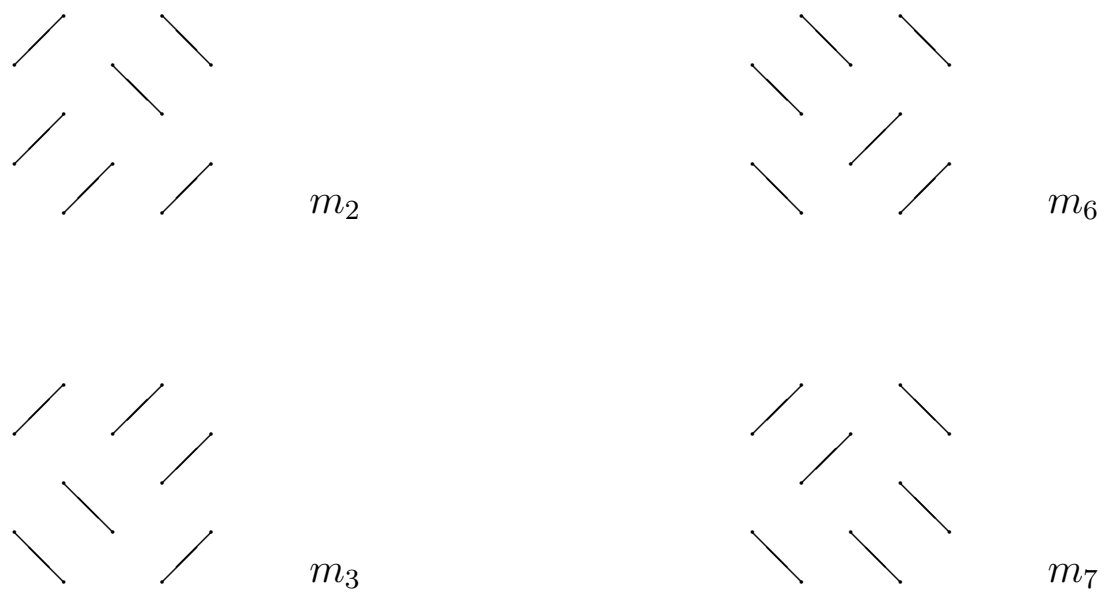

$m_{3}$
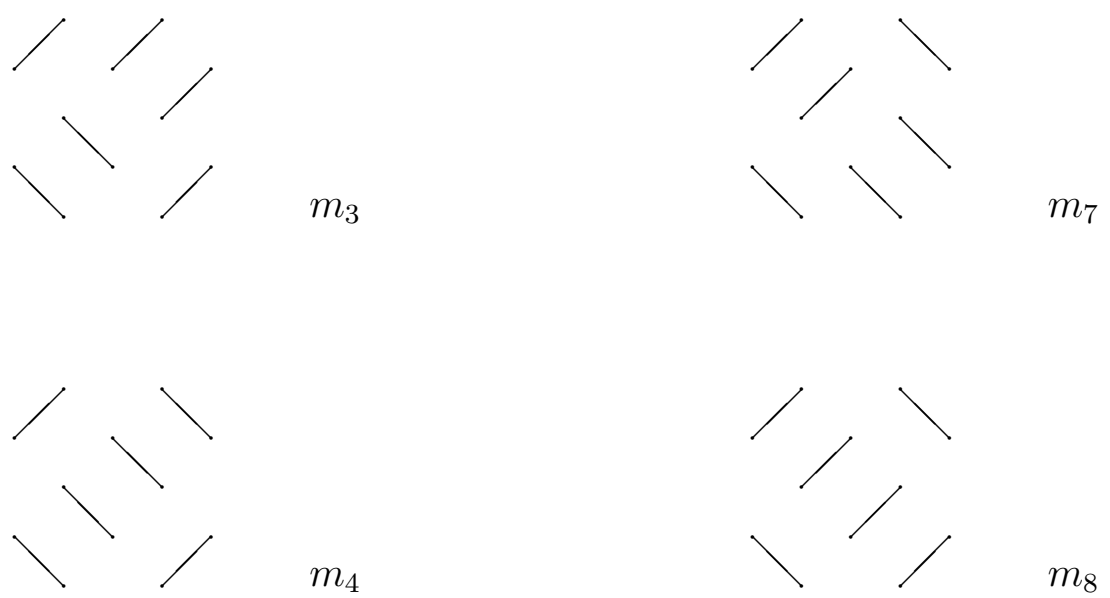

$m_{4}$

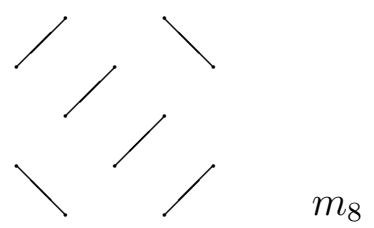

Figure 5: All matchings of the Aztec diamond graph of order 2 


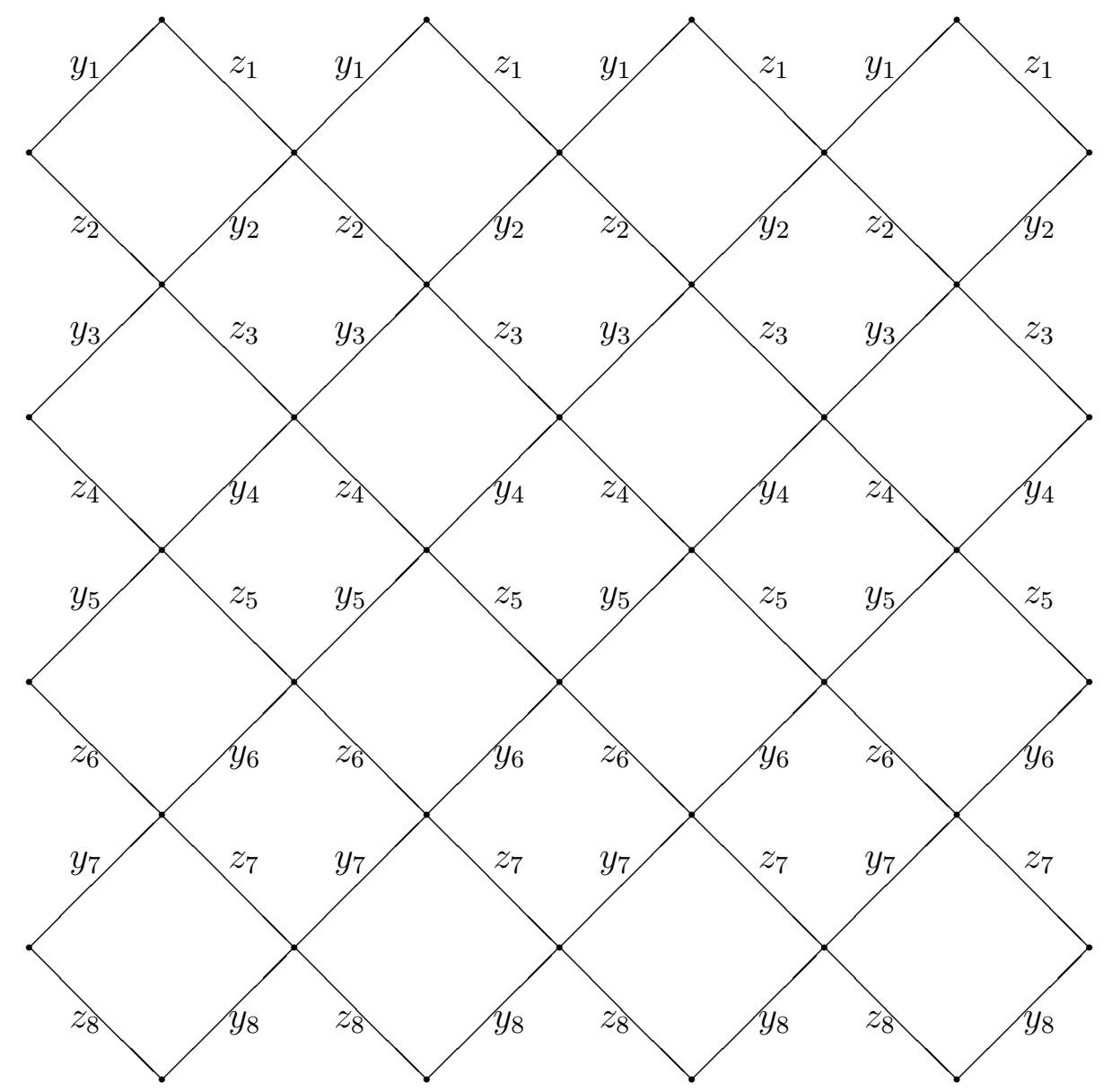

Figure 6: Aztec graph of order 4 with Stanley's weight labeling 


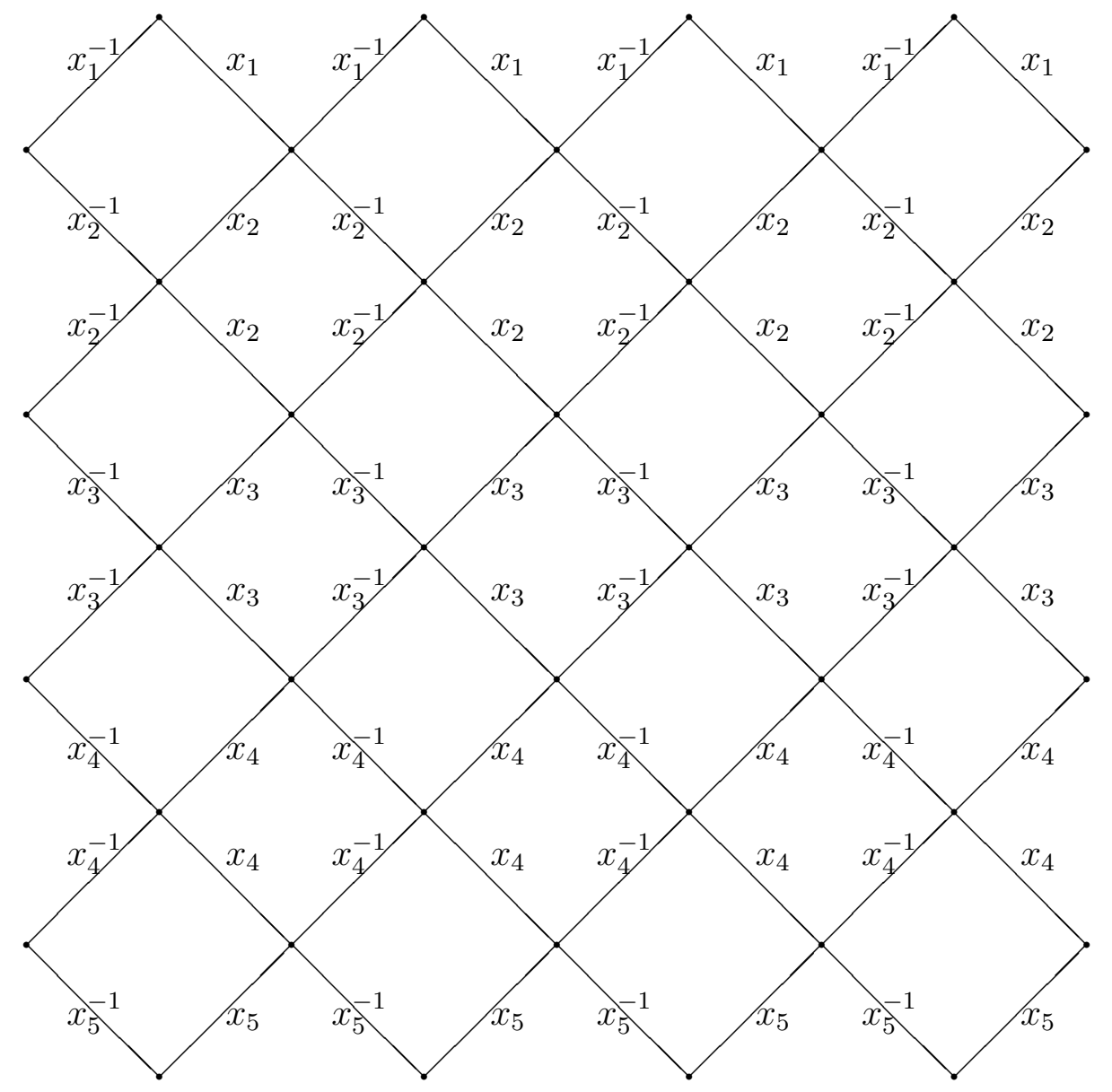

Figure 7: Aztec graph of order 4 with Weight Labeling A 


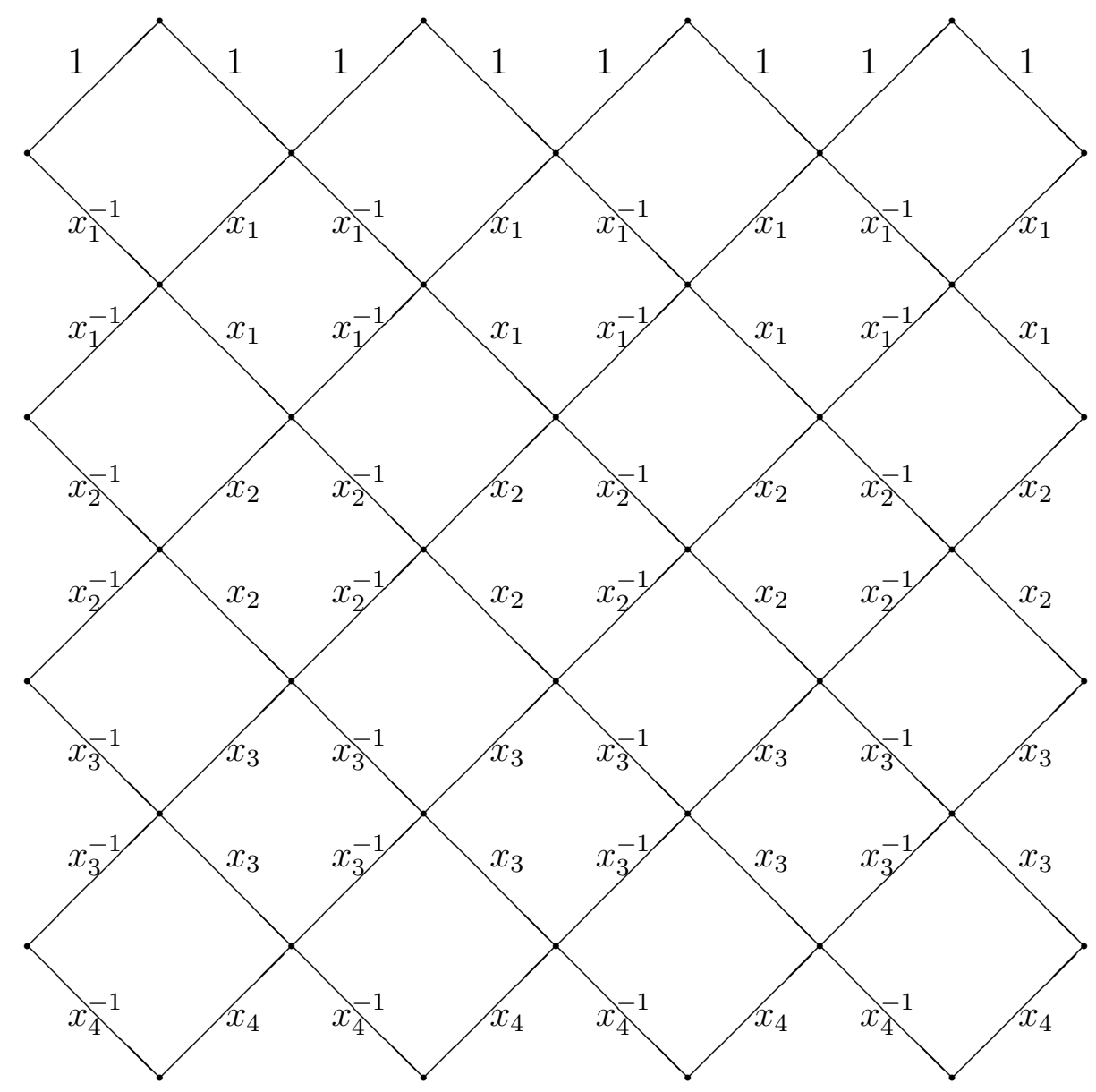

Figure 8: Aztec graph of order 4 with Weight Labeling B 


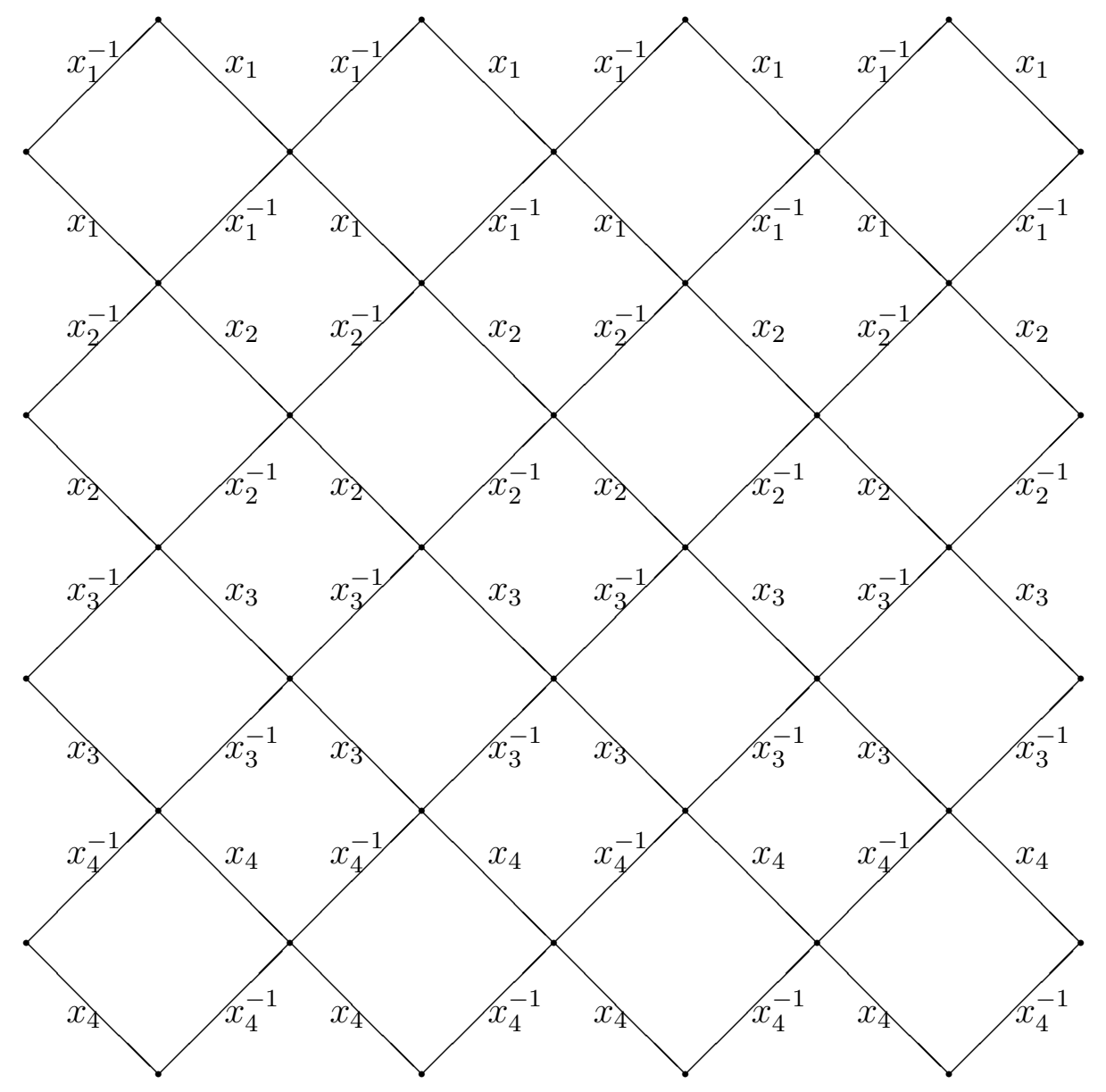

Figure 9: Aztec graph of order 4 with Weight Labeling C 


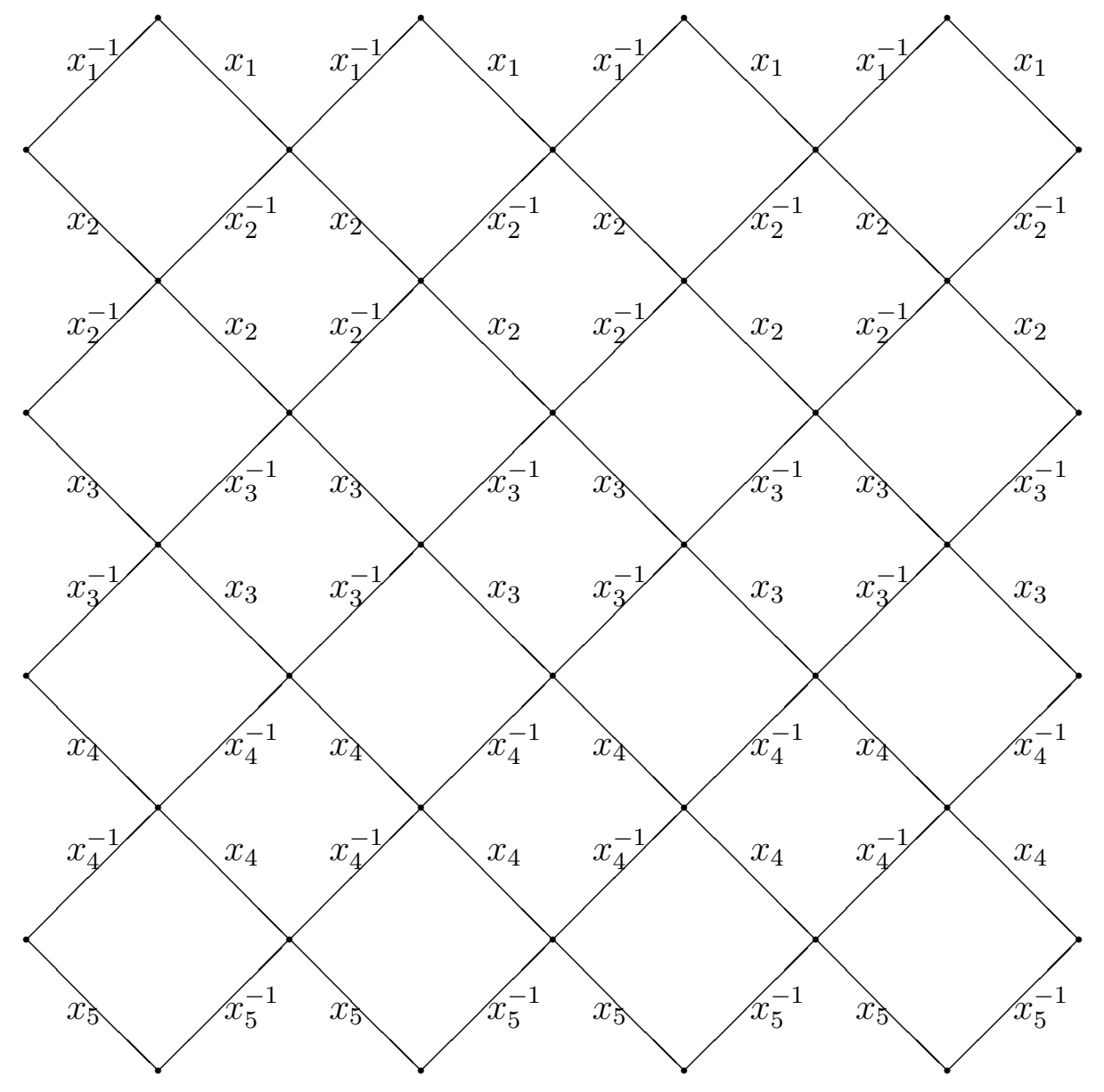

Figure 10: Aztec graph of order 4 with Weight Labeling D 


\begin{tabular}{ccc}
\hline Type & Number of Positive Roots & Positive Roots \\
\hline $\mathrm{A}_{n}$ & $\left(\begin{array}{c}n+1 \\
2\end{array}\right)$ & $\left\{\varepsilon_{i}-\varepsilon_{j} \mid 1 \leq i<j \leq n+1\right\}$ \\
$\mathrm{B}_{n}$ & $n^{2}$ & $\left\{\varepsilon_{i} \pm \varepsilon_{j} \mid 1 \leq i<j \leq n\right\} \bigcup\left\{\varepsilon_{i} \mid 1 \leq i \leq n\right\}$ \\
$\mathrm{C}_{n}$ & $n^{2}$ & $\left\{\varepsilon_{i} \pm \varepsilon_{j} \mid 1 \leq i<j \leq n\right\} \bigcup\left\{\varepsilon_{i} \mid 1 \leq i \leq n\right\}$ \\
$\mathrm{D}_{n}$ & $n(n-1)$ & $\left\{\varepsilon_{i} \pm \varepsilon_{j} \mid 1 \leq i<j \leq n\right\}$ \\
\hline
\end{tabular}

Figure 11: Positive Roots

\begin{tabular}{cccc}
\hline Type & Formula & Description of P & Description of Q \\
\hline $\mathrm{A}_{n}$ & $\mathrm{Ch}\left(\Psi_{\rho}\right)=\varpi(P)$ & Order $n$ \\
& & Weight Labeling A \\
\hline $\mathrm{B}_{n}$ & $\mathrm{Ch}\left(\Psi_{\rho}\right)=\varpi(P) \varpi(Q)$ & Order $n$ & Order $n-1$ \\
& & Weight Labeling B & Weight Labeling D \\
\hline $\mathrm{C}_{n}$ & $\mathrm{Ch}\left(\Psi_{\rho}\right)=\varpi(P) \varpi(Q)$ & Order $n$ & Order $n-1$ \\
& & Weight Labeling C & Weight Labeling A \\
\hline $\mathrm{D}_{n}$ & $\mathrm{Ch}\left(\Psi_{\rho}\right)=\varpi(P) \varpi(Q)$ & Order $n-1$ & Order $n-1$ \\
& & Weight Labeling D & Weight Labeling A \\
\hline
\end{tabular}

Figure 12: Summary of the theorems 


\section{References}

[B] N. Bourbaki, Groupes et Algèbres de Lie, Chapitres IV - VI, Hermann-Paris (1960).

[Br] D.M. Bressoud, Proofs and Confirmations, Cambridge Univ. Press, Cambridge U.K. 1999.

[C1] M. Ciucu, "Perfect matchings of cellular graphs," J. Algebraic Combin. 5 (1996), $87-103$.

[C2] M. Ciucu, "Perfect matchings and perfect powers," J. Algebraic Combin. 17 (2003), 335-375.

[EKLP1] N. Elkies, G. Kuperberg, M. Larsen, and J. Propp, "Alternating-sign matrices and domino tilings (Part I)," J. Algebraic Combin. 1 (1992), 111-132.

[EKLP2] N. Elkies, G. Kuperberg, M. Larsen, and J. Propp, "Alternating-sign matrices and domino tilings (Part II)," J. Algebraic Combin. 1 (1992), 219-234.

[FH] W. Fulton and J. Harris, Representation Theory, Springer-Verlag, New York, NY, 1991.

[H] J.E. Humphreys, Introduction to Lie Algebras and Representation Theory Graduate Texts vol. 9, Springer-Verlag, New York, NY, 1972.

$[\mathrm{K}] \quad$ E. Kuo, "Applications of graphical condensation for enumerating matchings and tilings," arXiv:math.CO/0304090.

[P] J. Propp, Talk, American Mathematical Society Meeting, San Diego, CA, Jan. 1997.

[P2] J. Propp, "Generalized domino shuffling," arXiv:math.CO/0111034.

[Y] B-Y. Yang, "Three Enumerations Problems Concerning Aztec Diamonds," Ph.D. thesis, Department of Mathematics, Massachusetts Institute of Technology, Cambridge, MA, 1991. 